\title{
Assessment of vocal fold mobility using dynamic magnetic resonance imaging and ultrasound in healthy volunteers
}

\author{
Magdalena Derlatka-Kochel', Pawel Kumoniewski , Marcin Majos ${ }^{3}$, Kamil Ludwisiak', Lech Pomorski², \\ Agata Majos ${ }^{1}$
}

'Department of Radiological, Isotopic Diagnosis and Therapy, Medical University of Lodz, Poland 2Department of General and Oncological Surgery, Chair of Surgical Clinical Sciences, Medical University of Lodz, Poland ${ }^{3}$ Department of Radiology and Diagnostic Imaging, Medical University of Lodz, Poland

\section{Abstract}

Purpose: To verify the value of dynamic magnetic resonance imaging (MRI) sequences, fast field echo (FFE), and balanced gradient echo (true fast imaging with steady-state free precession - TRUFI) in the evaluation of vocal fold mobility in healthy volunteers, against ultrasound examination (US) as the reference test.

Material and methods: Vocal fold mobility in 35 healthy volunteers (age 20-59 years, 20 women and 15 men) with no history of laryngeal disorders and neck surgeries was determined by means of US and MRI during normal breathing and phonation of the "hiiii" sound. US images were used to determine the glottic angles. During MRI two dynamic sequences, fast field echo and balanced gradient echo, were applied to determine the minimum and maximum values of the glottic angles, along with the rima glottidis area, separately for the right and left compartments. Due to differences in larynx anatomy, the abovementioned parameters were analysed separately for women and men.

Results: No significant differences were observed between the glottic angle values obtained during US and dynamic MRI (FFE and TRUFI sequences). Regardless of the dynamic MRI sequence used, a positive correlation was found between the maximum values of glottic angle and the rima glottidis area. This correlation was strong and statistically significant among men, but not in women.

Conclusions: Dynamic MRI of vocal folds using FFE and TRUFI sequence is an accurate method for the objective evaluation of rima glottidis width.

Key words: ultrasound, mobility, magnetic resonance, vocal cords, vocal folds.

\section{Introduction}

Vocal folds are paired mobile anatomical structures located at the lateral internal walls of the larynx, which play a significant role in voice emission. During regular breathing, vocal folds are abducted, which results in widening of the rima glottidis. During phonation, the folds are adducted and oscillate.

Currently, videolaryngoscopy is the test of choice in the evaluation of vocal-fold mobility disorders. However, videolaryngoscopy is an invasive and expensive method that requires dedicated and not necessarily widely available equipment, causes various degrees of discomfort in patients, and necessitates their good cooperation with the examiner. Furthermore, the quality of the result is to a large degree dependent on anatomical conditions, as well as the operator's experience. Another drawback of videolaryngoscopy is the difficulty of obtaining objective measurements of the rima glottidis [1].

The list of imaging studies that are suitable for the evaluation of vocal folds includes ultrasonography (US), computed tomography $(\mathrm{CT})$, and magnetic resonance imaging

Correspondence address:

Marcin Majos, Department of Radiology and Diagnostic Imaging, Medical University of Lodz, 251 Pomorska St., $92-213$ Łódź, Poland,

e-mail: marcin.majos@stud.umed.lodz.pl

Authors' contribution:

A Study design - B Data collection - C Statistical analysis - D Data interpretation - E Manuscript preparation - F Literature search - G Funds collection 
(MRI) [2-4].While published data confirm the usefulness of all these methods, ultrasonography and MRI seem to be more appropriate for vocal fold evaluation because they are not associated with exposure to ionising radiation and, even more importantly, are suitable for dynamic imaging. To date, US is considered the reference test in vocal fold examination. However, because of advantages in MRI techniques, such as shorter acquisition time, better availability, excellent tissue resolution, and most of all, due to a substantial increase in the number of indications to MRI in patients with pathologies of the neck, the evaluation of this method's usefulness in the examination of rima glottidis has become a vital issue.

The aim of this study was to verify the value of dynamic MRI sequences, fast field echo (FFE, Siemens) and balanced gradient echo (true fast imaging with steadystate free precession, TRUFI, Siemens) in the evaluation of vocal fold mobility in healthy volunteers, against US as the reference test.

\section{Material and methods}

The protocol of the study was approved by the Local Bioethics Committee at the Medical University of Lodz (decision no. RNN/187/18/KE of 15 May 2018). The study included 35 healthy volunteers ( 20 women and 15 men) aged between 20 and 59 years, with no history of dysphonia or upper respiratory disorders other than upper respiratory tract infections. After expressing their written informed consent to participate in the study, the volunteers were instructed how to cooperate with the examiner during data acquisition. Before the vocal fold imaging, all participants of the study underwent a basic laryngological examination.

Aside from the vocal folds, the US and MRI assessment included also the evaluation of all other organs of the neck, among them thyroid and salivary glands, and lymph nodes. None of the study participants presented with any pathologies within these organs.

Vocal fold mobility was observed during normal breathing and phonation. During both US and MRI, the participants were instructed to produce a prespecified test sound after hearing the "start" command, and to continue until the "stop" command. The test sound was defined as "hiii" because phonation of the "i" vowel sound is known to be associated with maximum narrowing of the rima glottidis [5].

The position of vocal folds depends on the respiration phase, with the rima glottidis being wider on inspiration and narrower on expiration [6]. Minimum values of the angles and areas were determined during maximum adduction of vocal folds, and the maximum values during their maximum adduction during normal breathing.

\section{Ultrasonography}

Ultrasonography examinations were carried out with a Logiq S8 ultrasonograph (GE Healthcare) equipped with an ML6-15 (5-13 MHz) linear probe with $10 \mathrm{MHz}$ frequency. The examination was conducted with the participant in a supine position, with the head tilted backwards. The probe was applied transversely to the long axis of the body, in the midline at the level corresponding to the mid-segment of the thyroid cartilage. Then, the probe was advanced along the long axis to identify a position in which both vocal folds could be visualised symmetrically.

The outcomes of the examination, in the form of static images and videos, were archived onto memory drives. The images were used to verify whether a complete closure of the glottis took place during the phonation (Figure 1). Furthermore, the images documenting maximum and minimum deviation of vocal folds from the midline were selected to determine the minimum and maximum glottic angles for the right and left compartment of the rima glottidis with the aid of ImageJ software [7] (https://imagej. nih.gov/ij/) (Figure 2).

\section{Magnetic resonance imaging}

MRI was carried out with 1.5T Magnetom Avanto scanner (Siemens) using an eight-channel neck coil and a four-channel head coil. No contrast agents were administered. Static T2-weighted sequences, turbo spin echo (TSE) in the axial plane, and inversion recovery (IR; turbo inversion recovery magnitude - TIRM, Siemens) in

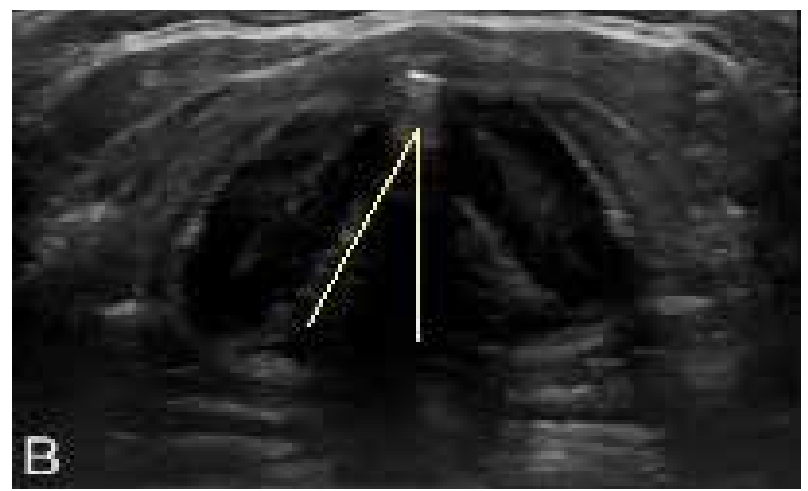

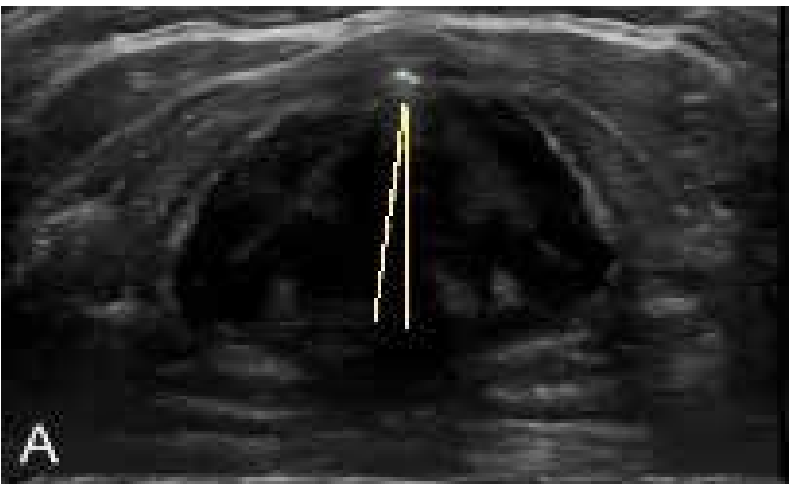

Figure 1. Ultrasonography - measurement of the minimum (A) and maximum (B) angle between the vocal fold and the midline during normal breathing 
the frontal plane were obtained along with the dynamic sequences FFE and TRUFI in the axial plane. Detailed information about the MRI protocol is presented in Table 1 .

Based on pilot sagittal images, the coronal T2-weighted sequence was oriented perpendicularly to the horizontal plane of both vocal folds (Figure 3). Then, one of the coronal slices was chosen to determine the level at which the vocal folds were later visualised during dynamic studies.

Abduction and adduction of vocal folds during normal breathing and phonation were evaluated using dynamic gradient sequences FFE and TRUFI (Figure 4). In the case of FFE sequences, three transverse slices at the larynx level were obtained during each acquisition. The sequences were oriented in such a way that the rima glottidis was on the central slice, and the remaining two slices contained the epiglottic and subglottic compartments. Using this approach, three acquisitions were carried out during phonation, followed by another three acquisitions during normal breathing, with a total of 15 acquisitions, corresponding to a total of 45 slices.

In the case of TRUFI sequences, 60 transverse slices were obtained, all at the same level. The first 20 slices were obtained during phonation and the remaining ones during normal breathing.

The slices were analysed with software provided by the scanner's manufacturer (Syngo.via, Siemens). The area of the rima glottidis was measured using the ROI freehand function. First, a line connecting the anterior and posterior commissure and dividing the rima glottidis into two symmetric compartments, the right and the left one, was defined. Then, the right and left glottic angles between the midline and the right and left vocal fold, respectively, were measured along with the areas of the right and left rima glottidis compartments defined as the areas between the midline and respective vocal folds (Figure 5).

Statistical analysis of the results was carried out with STATISTICA software (StatSoft, USA). Statistical characteristics of analysed variables were presented as arithmetic means, standard deviations, and medians. Normal distribution of the study variables was verified with the

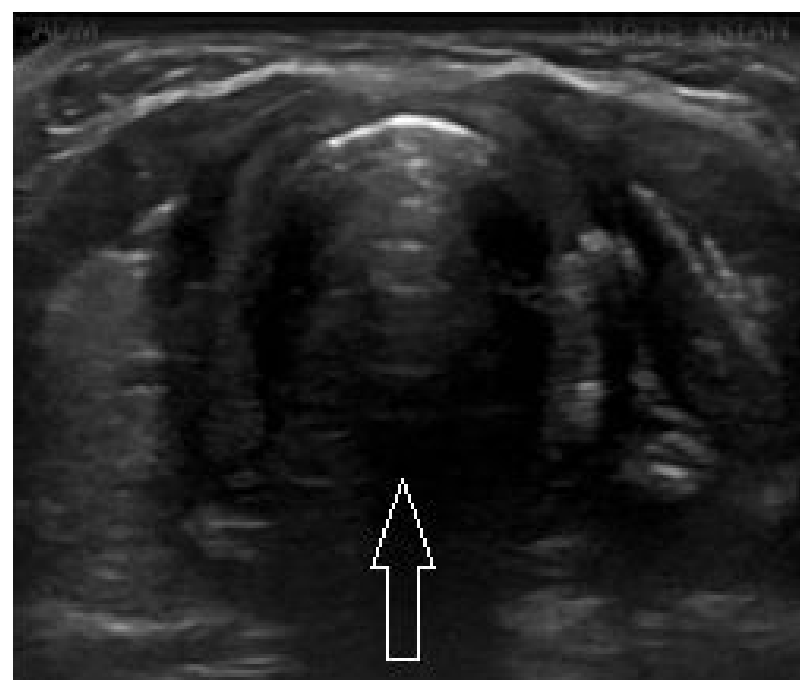

Figure 2. Ultrasonography - vocal folds during voice emission

Shapiro-Wilk test. The variables with distributions other than normal were analysed with Friedman's test with Dunn's post-hoc tests or Kruskal-Wallis test. The results were considered significant for $p$-values $<0.05$.

\section{Results}

Regardless of the imaging method, symmetric, uninterrupted mobility of vocal folds could be observed during normal breathing, and complete closure of the rima glottidis during phonation was documented in all patients.

Mean values of minimum and maximum glottic angles determined using US and dynamic MRI during normal breathing did not differ significantly, either for the whole study group or for women and men separately. All $p$-values determined on Dunn's test were higher than 0.05 . Detailed results are shown in Table 2.

Furthermore, no statistically significant differences were found between the two types of dynamic MRI sequences in the measurement of the rima glottidis area during normal breathing, either for the whole study group or after stratifying the results according to participants' sex.

Table 1. Parameters of obtained dynamic and static sequences

\begin{tabular}{|l|c|c|c|c|}
\hline \multirow{2}{*}{ Type of sequence } & \multicolumn{2}{|c|}{ Dynamic examination } & \multicolumn{2}{c|}{ Static examination } \\
\cline { 2 - 5 } & FFE & Balanced GE (TRUFI) & IR (TIRM) & 3600 \\
\hline Repetition time (ms) & 23 & 364.76 & 7640 & 82 \\
\hline Echo time (ms) & 3.64 & 1.29 & $0.9 \times 0.9$ & $0.8 \times 0.8$ \\
\hline Image resolution & $1.1 \times 1.1$ & $1.4 \times 1.1$ & 3 & 4 \\
\hline Slice thickness & 6 & 10 & 25 & 15 \\
\hline Number of slices & 3 & 1 & $220 \times 220$ & $210 \times 210$ \\
\hline Field of view (m) & 20 & 62 & $4: 42$ & $3: 43$ \\
\hline Total acquisition time & $220 \times 220$ & $280 \times 280$ & Not applicable & Not applicable \\
\hline Number of dynamic scans & $1: 08$ & $0: 22$ & & \\
\hline
\end{tabular}



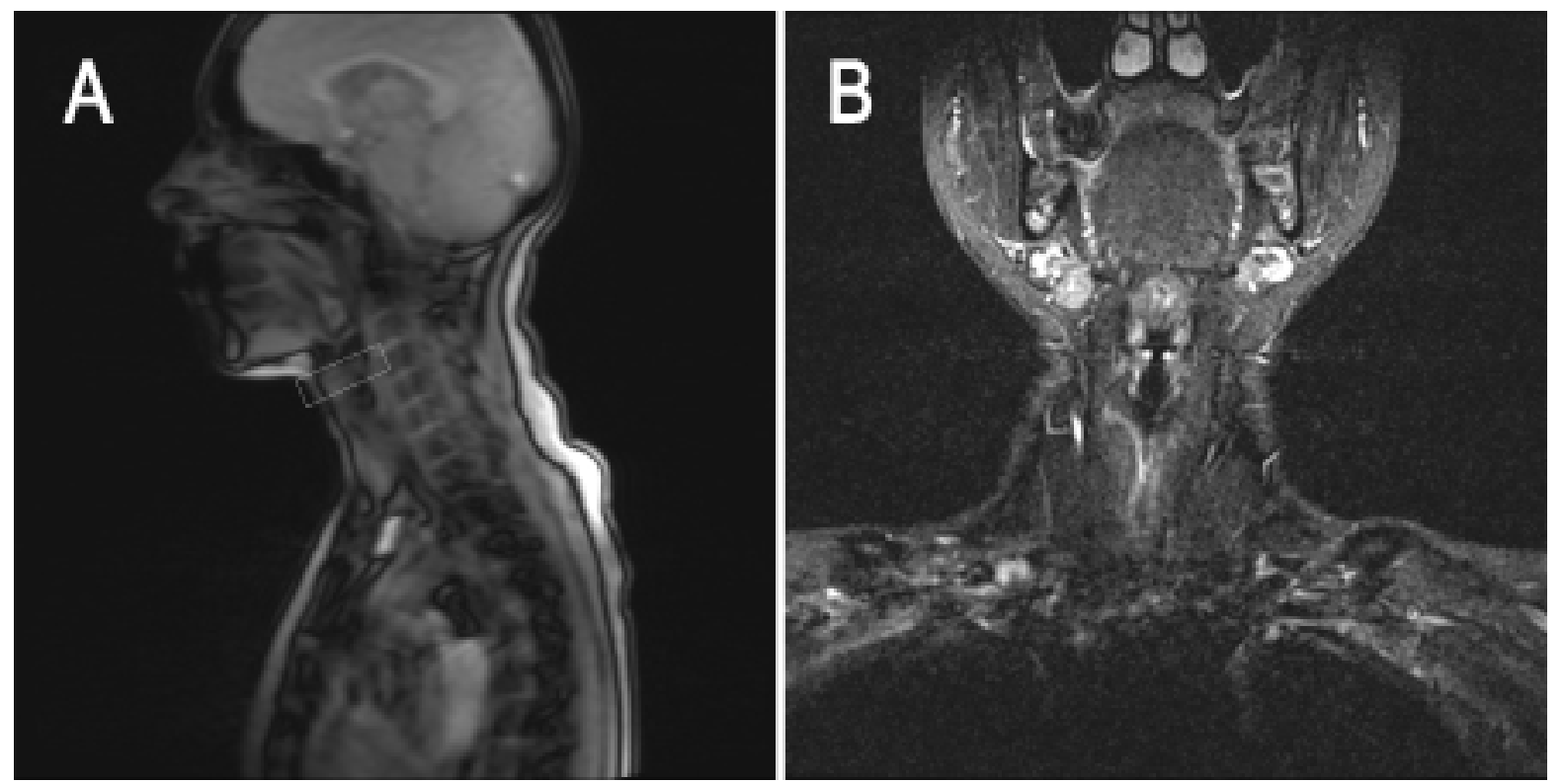

Figure 3. A) Pilot sagittal sequence. B) Coronal turbo inversion recovery magnitude (TIRM) sequence. Identification of a plane for dynamic magnetic resonance imaging
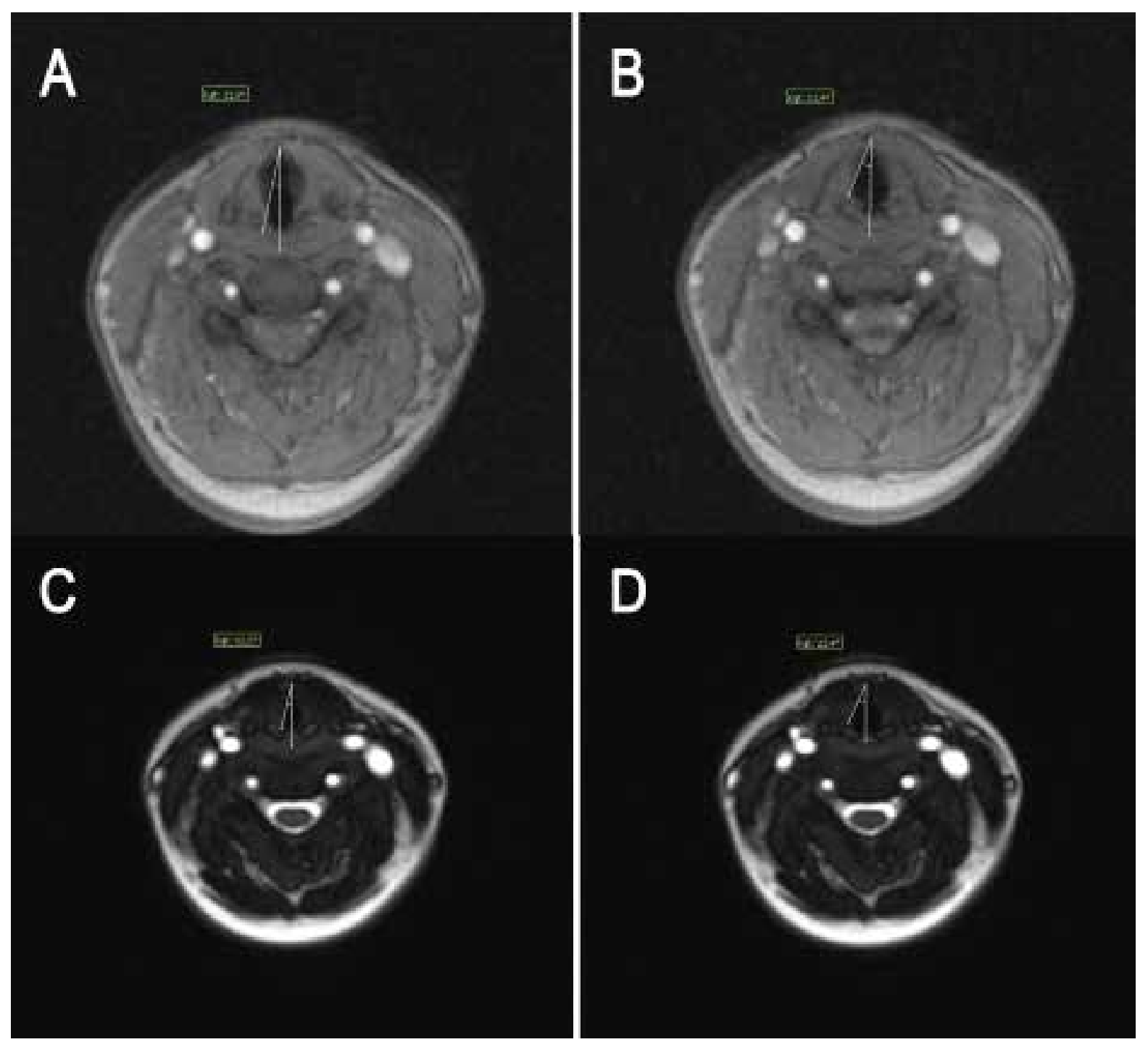

Figure 4. Rima glottidis on dynamic magnetic resonance imaging sequences: A-B) fast field echo (FFE) and C-D) true fast imaging with steady-state free precession (TRUFI) during normal breathing 

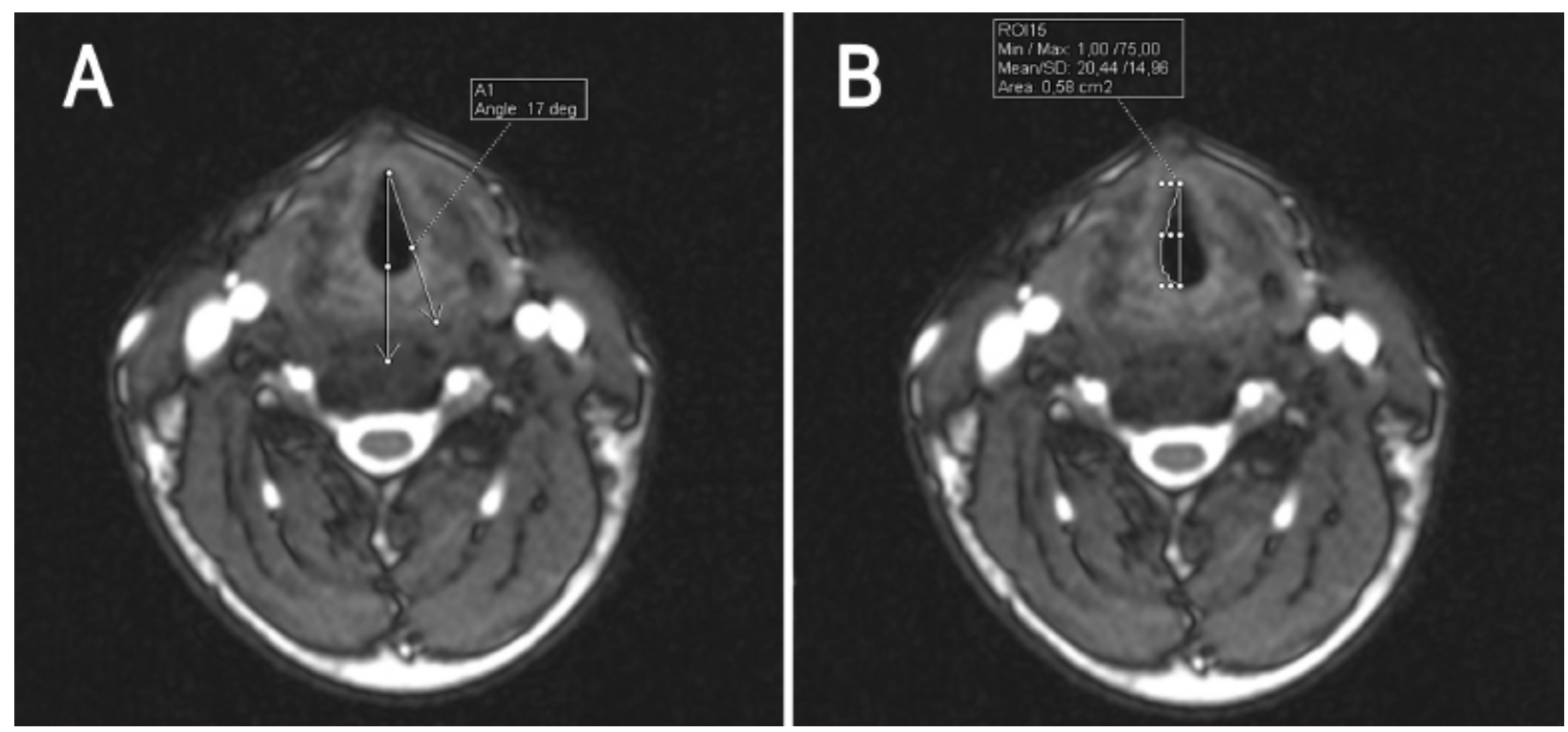

Figure 5. Dynamic true fast imaging with steady-state free precession (TRUFI) sequence, axial slices. Representative measurements of the glottic angle (A) and rima glottidis area (B)

Table 2. Mean values of minimum and maximum glottic angles during normal breathing

\begin{tabular}{|l|c|c|c|c|c|c|}
\hline \multirow{2}{*}{ Parameter } & \multicolumn{3}{|c|}{ Minimum angle $\left(^{\circ}\right)$} & \multicolumn{3}{c|}{ Maximum angle $\left(^{\circ}\right)$} \\
\cline { 2 - 7 } Method & USG & FFE MRI & TRUFI MRI & USG & FFE MRI & TRUFI MRI \\
& $7.17 \pm 1.69$ & $9.54 \pm 3.18$ & $8.11 \pm 2.14$ & $17.09 \pm 4.00$ & $16.63 \pm 4.46$ & $17.46 \pm 3.73$ \\
& $(\mathrm{Me}=7)$ & $(\mathrm{Me}=8)$ & $(\mathrm{Me}=8)$ & $(\mathrm{Me}=17)$ & $(\mathrm{Me}=16)$ & $(\mathrm{Me}=18)$ \\
\hline Women & $7.35 \pm 1.57(\mathrm{Me}$ & $9.55 \pm 3.36$ & $7.95 \pm 2.21$ & $17.00 \pm 3.85$ & $16.95 \pm 4.05$ & $17.95 \pm 3.17$ \\
& $=7)$ & $(\mathrm{Me}=9)$ & $(\mathrm{Me}=8)$ & $(\mathrm{Me}=17)$ & $(\mathrm{Me}=17)$ & $(\mathrm{Me}=18)$ \\
\hline Men & $6.93 \pm 1.87$ & $9.53 \pm 3.02$ & $8.33 \pm 2.09$ & $17.20 \pm 4.33$ & $16.20 \pm 5.07$ & $16.80 \pm 4.40$ \\
& $(\mathrm{Me}=7)$ & $(\mathrm{Me}=8)$ & $(\mathrm{Me}=8)$ & $(\mathrm{Me}=16)$ & $(\mathrm{Me}=15)$ & $(\mathrm{Me})=15)$ \\
\hline
\end{tabular}

Table 3. Mean values of minimum and maximum rima glottidis area during normal breathing determined based on dynamic magnetic resonance imaging sequences

\begin{tabular}{l|c|c|c|c|}
\multirow{2}{*}{$\begin{array}{l}\text { Parameter } \\
\text { Sequence }\end{array}$} & \multicolumn{2}{|c|}{ Minimum area $\left(\mathrm{cm}^{2}\right)$} & \multicolumn{2}{c|}{ Maximum area $\left(\mathrm{cm}^{2}\right)$} \\
\cline { 2 - 5 } Whole group & $0.36 \pm 0.15(\mathrm{Me}=0.30)$ & $0.26 \pm 0.11(\mathrm{Me}=0.25)$ & $0.62 \pm 0.21(\mathrm{Me}=0.60)$ & $0.67 \pm 0.20(\mathrm{Me}=0.60)$ \\
\hline Women & $0.23 \pm 0.08(\mathrm{Me}=0.30)$ & $0.20 \pm 0.09(\mathrm{Me}=0.20)$ & $0.48 \pm 0.10(\mathrm{Me}=0.50)$ & $0.56 \pm 0.16(\mathrm{Me}=0.53)$ \\
\hline Men & $0.47 \pm 0.15(\mathrm{Me}=0.45)$ & $0.34 \pm 0.10(\mathrm{Me}=0.35)$ & $0.81 \pm 0.17(\mathrm{Me}=0.80)$ & $0.78 \pm 0.19(\mathrm{Me}=0.80)$ \\
\hline
\end{tabular}

The $p$-values obtained with the Kruskal-Wallis test were higher than 0.05. Mean rima glottidis areas determined with both methods during normal breathing are presented in Table 3.

Regardless of the type of MRI sequences, a positive correlation was found between maximum glottic angle and maximum rima glottidis area. The coefficients of correlation were 0.355 and $0.541(p<0.05)$ for FFE sequences in women and men, respectively, and 0.232 and $0.516(p<0.05)$ for TRUFI sequences in women and men, respectively. The correlation between maximum glottic angle and rima glottidis area was strong and statistically significant only in men.

\section{Discussion}

Assessment of vocal fold mobility seems to be particularly interesting from a clinical perspective, either in the evaluation of vocal fold paralysis or in the determination of the true extent of inflammatory and proliferative processes involving adjacent tissues. Vocal fold mobility assessment might also be a valuable instrument in the qualification for surgical treatment, as well as in the detection and monitoring of surgical complications in patients operated for multiple conditions involving the neck and chest. 
Observation of vocal fold mobility is an essential component of the laryngoscopic evaluation. While laryngoscopy can provide quantitative data about the size of the rima glottidis, it is technically challenging. This stimulated research on alternative methods for an objective, i.e. reproducible and accurate measurement of vocal folds and rima glottidis. Without doubt, the group of such methods also includes imaging studies, US, and MRI.

Transcutaneous US is a non-invasive atraumatic examination suitable for objective assessment of vocal fold mobility in real time [8]. The usefulness of this method and its high sensitivity have already been demonstrated in previous studies $[4,9,10]$, and thus, US is considered an alternative to laryngoscopy, especially during the perioperative assessment of patients with goitre or thoracic aortic aneurysms [11]. The limitations of US include the inability to obtain diagnostic images in patients with calcifications of the thyroid cartilage (more often found in men), as well as problems with the evaluation of patients with higher BMI, older age, male sex, or those after neck surgery $[12,13]$. Transcutaneous US of vocal folds is not a routine examination. Usually, this examination is carried out in centres with well-developed departments of endocrinological surgery.

Similar to US, the primary advantages of MRI include its non-invasive character and lack of exposure to ionising radiation. Among a plethora of available MRI sequences, the most suitable are those enabling dynamic real-time imaging with an optimal spatial resolution and short acquisition time [14]. MRI is also suitable for objective measurement of the rima glottidis. This is particularly important in the case of patients who are not eligible for laryngoscopy and US, e.g. due to anatomical obstacles such as tumours, scar tissue, or massive calcifications of the thyroid cartilage [15]. Furthermore, it should be emphasised that MRI provides the highest tissue resolution of all available imaging studies.

Nevertheless, MRI has also some drawbacks, including its relatively high cost and limited availability. While the interpretation of MRI results can be hindered by motor artefacts associated with the pulsation of blood vessels, swallowing, or coughing, their occurrence has been substantially reduced due to technological advances, such as breathing sensors, dedicated larynx coils, and new, fast sequences $[14,16]$.

An important factor determining the quality of dynamic imaging of the larynx is an appropriate selection of the imaging plane. Transverse slices should be precisely parallel to the vocal folds. In our present study, the level of visualisation was determined based on the analysis of coronal slices. In some patients, no distinct separation of vocal folds was observed on coronal images. Planning a study, one should consider changes in the position of the larynx in the sagittal plane during phonation [5]. However, none of the conditions mentioned above seem to be a significant limitation of dynamic MRI. Due to ultrashort acquisition time, in the case of the sequences used in this study $22 \mathrm{~s}$ or $68 \mathrm{~s}$, the dynamic scans can be repeated a virtually unlimited number of times, until adequate slices are obtained in a time regimen that is comfortable for the patient.

Only a few previous studies have dealt with dynamic MRI of the vocal folds. Schlamman and Faust confirmed consistency of results obtained by means of MRI with a 1.5T scanner and laryngoscopy, but their observations were based solely on qualitative analysis. Ahmad et al. analysed changes in the position of vocal folds in transverse and sagittal slices obtained with a $1.5 \mathrm{~T}$ scanner during phonation of various sounds by healthy volunteers. Those authors were the first to estimate the width of the rima glottidis on the basis of the angle between both vocal folds [5]. Baki et al. used a 3.0T scanner to examine healthy persons and patients with unilateral vocal fold paralysis [3]. Based on MRI images, those authors determined the angle between each vocal fold and the midline and calculated the area of the right and left rima glottidis compartment. We used the same methodology in our present study, but, importantly, the images were obtained with a $1.5 \mathrm{~T}$ scanner. Hence, we confirmed that dynamic imaging of vocal folds is possible with the most widely available scanners, and as such, can be used in routine clinical practice.

Moreover, the protocol of our study included two dynamic sequences not used by Baki et al.: FFE and TRUFI, the former already positively verified by those authors for a 3.0T scanner [3]. This sequence was shown to be suitable for reliable assessment of vocal fold abduction and adduction, as well as for the diagnostics of vocal fold paralysis. In the present study, the FFE sequence was accommodated to a 1.5T scanner, which enabled us to acquire a total of 15 slices at three levels within an acquisition time of 68 seconds. An advantage of this sequence is the possibility of obtaining three transverse scans at various levels simultaneously, which makes it applicable for the examination of patients with more complex anatomical conditions and/or greater phonatory mobility of the epiglottis in the sagittal plane.

Another dynamic sequence analysed in our study was TRUFI, which is characterised by the highest image to time ratio, 60 scans per 22 seconds. Previous studies demonstrated high effectiveness of this sequence in dynamic imaging of hand movements, albeit with a 3.0T scanner [17]. The slices obtained with this sequence accurately depict even subtle changes in fast-moving anatomical structures.

The primary aim of this study was to compare the results obtained using dynamic MRI sequences with the outcomes of transcutaneous US, which is currently the gold standard in vocal fold assessment. We compared these two methods based on the values of glottic angles formed by the vocal folds and the midline during normal breathing. The measurements were taken separately for the right and left rima glottidis compartments. Vocal-fold mobility disorders can be unilateral or bilateral, and hence only the 
approach described above can accurately reflect the actual clinical situation. Moreover, the results were stratified according to patient sex [7]. Regardless of the analysed MRI sequence, the values of glottic angles did not differ significantly from those obtained using US; this implies that dynamic MRI is an accurate method for vocal fold assessment.

We also analysed a correlation between the maximum glottic angle and maximum rima glottidis area. These two parameters correlated positively with each other, but a significant strong correlation was observed only among men (0.541 for FFE and 0.516 for TRUFI), whereas the relationship in women was weak and insignificant $(0.355$ for FFE and 0.232 for TRUFI). This discrepancy was probably a consequence of sex-specific differences in the anatomical structure of the larynx. Thus, our findings suggest that the rima glottidis area and the glottic angle can be used interchangeably as the indices of rima glottidis morphology in men, but these parameters should be interpreted carefully in women. Further studies involving a substantially larger population of volunteers are needed to verify the correlations identified herein in an entirely objective manner. Another objective of future research, important from a clinical perspective, would be the identification of the reference values for the glottic angles and rima glottidis area in women and men.
Despite similarities in the methodology of both studies, direct comparison of our findings with the results published by Baki et al. is not possible because the latter were presented in a complex manner. Instead of absolute values, those authors reported relative changes in the rima glottidis area and glottic angle during phonation. In our opinion, determination of the absolute values, although it might be considered an oversimplification by some, is more useful from a clinical perspective as a simple, rapid, and effective method for the vocal fold mobility assessment.

\section{Conclusions}

Dynamic MRI using GRE and balanced GE sequences might be an accurate method for objective assessment of vocal fold mobility, helpful in the diagnostics of neck pathologies, qualification for surgical procedures, and monitoring of treatment outcomes. Dynamic sequences should be included in the standard MRI protocols for neck examination.

\section{Conflict of interest}

The authors report no conflict of interest.

\section{References}

1. Dailey SH, Kobler JB, Hillman RE, et al. Endoscopic measurement of vocal fold movement during adduction and abduction. Laryngoscope 2005; 115: 178-183.

2. Kim BS, Ahn KJ, Park YH, et al. Usefulness of laryngeal phonation CT in the diagnosis of vocal cord paralysis. Am J Roentgenol 2008; 190: 1376-1379.

3. Baki MM, Menys A, Atkinson D, et al. Feasibility of vocal fold abduction and adduction assessment using cine-MRI. Eur Radiol 2017; 27: 598-606.

4. Wong $\mathrm{KP}, \mathrm{BLang} \mathrm{BHH}, \mathrm{Ng} \mathrm{SH}$, et al. A prospective, assessor-blind evaluation of surgeon-performed transcutaneous laryngeal ultrasonography in vocal cord examination before and after thyroidectomy. Surgery 2013; 154: 1158-1165.

5. Ahmad M, Dargaud J, Morin A, et al. Dynamic MRI of larynx and vocal fold vibrations in normal phonation. J Voice 2009; 23: 235-239.

6. Brancatisano T, Collett PW, Engel LA. Respiratory movements of the vocal cords. J Appl Physiol 1983; 54: 1269-1276.

7. Rueden CT, Schindelin J, Hiner MC, et al. ImageJ2: ImageJ for the next generation of scientific image data. BMC Bioinformatics 2017; 18: $1-26$.

8. Hu Q, Zhu SY, Luo F, et al. High-frequency sonographic measurements of true and false vocal cords. J Ultrasound Med 2010; 29: 1023-1030.

9. Brian KW, Shi HL, Au KL, et al. Determining the learning curve of transcutaneous laryngeal ultrasound in vocal cord assessment by CUSUM analysis of eight surgical residents: when to abandon laryngoscopy. World J Surg 2016; 40: 659-664.

10. Cheng SP, Lee JJ, Liu TP, et al. Preoperative ultrasonography assessment of vocal cord movement during thyroid and parathyroid surgery. World J Surg 2012; 36: 2509-2515.

11. Ali S, Athar M, Ahmed SM. Basics of CPB. Indian J Anaesth 2019; 49: 257-262.

12. Wong KP, Lang BHH, Chang YK, et al. Assessing the validity of transcutaneous laryngeal ultrasonography (TLUSG) after thyroidectomy: what factors matter? Ann Surg Oncol 2015; 22: 1774-1780.

13. Kandil E, Deniwar A, Noureldine SI, et al. Assessment of vocal fold function using transcutaneous laryngeal ultrasonography and flexible laryngoscopy. JAMA Otolaryngol Head Neck Surg 2016; 142: 74-78.

14. Ruytenberg T, Verbist BM, Vonk-Van Oosten J, et al. Improvements in high resolution laryngeal magnetic resonance imaging for preoperative transoral laser microsurgery and radiotherapy considerations in early lesions. Front Oncol 2018; 8: 216.

15. Aziz M. Advances in laryngoscopy. F1000Res 2015; 4: 1410.

16. Schlamann M, Lehnerdt G, Maderwald S, et al. Dynamic MRI of the vocal cords using phased-array coils: a feasibility study. Indian J Radiol Imaging 2009; 19: 127-131.

17. Bayer T, Adler W, Janka R, et al. Magnetic resonance cinematography of the fingers: a 3.0 Tesla feasibility study with comparison of incremental and continuous dynamic protocols. Skeletal Radiol 2017; 46: 1721-1728. 